\title{
Magnitude and energy of earthquakes
}

\author{
Beno Gutenberg and Charles Francis Richter
}

Annali di Geofisica, Vol. 9, n. 1, 1956.

\section{ABSTRACT}

Discrepancies arise among magnitudes as derived from local earthquake data $\left(\mathrm{M}_{L}\right)$, body waves $\left(\mathrm{M}_{\mathrm{B}}\right)$ and surface waves $\left(\mathrm{M}_{S}\right)$. The relation of $\mathrm{M}_{L}$ to the others is as yet not definitive; but $\mathrm{M}_{\mathrm{S}}-\mathrm{m}_{\mathrm{B}}=\mathrm{a}\left(\mathrm{M}_{\mathrm{S}}-\mathrm{b}\right)$. The latest revision gives $\mathrm{a}=0.37, \mathrm{~b}=6.76$. Pending further research it is recommended that $\mathrm{M}_{\mathrm{L}}$ continue to be used as heretofore, but $\mathrm{M}_{\mathrm{S}}$ (and ultimately $\mathrm{M}_{\mathrm{L}}$ ) should be referred to $\mathrm{m}_{\mathrm{B}}$ as a general standard, called the unified magnitude and denoted by $\mathrm{m}$. Tentatively $\log \mathrm{E}=5.8+2.4 \mathrm{~m}$ (E in ergs). Revised tables and charts for determining $m$ are given.

This paper is in continuation of previous investigations [Gutenberg and Richter 1942, 1956].

The earthquake magnitude has statistical and other uses independent of the relation between magnitude and energy. Indeed, it is possible that there is no complete one-to-one correlation between magnitude and energy for large and complex tectonic events. Even so, a mean or representative relation is a legitimate object of inquiry. In attempting to refine the magnitude-energy relation it was found [Gutenberg and Richter 1956] that three imperfectly consistent magnitude scales had been in use:

$M_{L}$ determined from records of local earthquakes according to the original definition [Richter 1935];

$M_{S}$ from the amplitudes of surface waves for shallow teleseisms, [Gutenberg and Richter 1936; Gutenberg 1945a];

$m_{B}$ from the amplitude/period ratio of body waves for teleseisms, shallow and deep-focus [Gutenberg 1945b, 1945c].

The two latter were originally adjusted to coincide near $M=7$, but were later found to diverge linearly so that

$$
M_{S}-m_{B}=\mathrm{a}\left(M_{S}-b\right)
$$

For a number of years reductions were carried out with $a=1 / 4, b=7$, converting values of $m_{B}$ into the corresponding $M_{S}$. The result of this reduction may be designated $M_{B}$. The final value given for $M$ was a weighted mean between $M_{B}$ and $M_{S}$. This may be taken as defining $M$ without subscript. The adjustment between $M_{S}$ and $M_{B}$ can now be performed with considerable accuracy, using the relation (1) with revised parameters $a=0.37, b=6.76$.
This is equivalent to

$$
m_{B}=0.63 M_{S}+2.5=M_{S}-0.37\left(M_{S}-6.76\right)
$$

The revision is based on a large body of data. Magnitudes have been derived by the senior author, from surface waves and from body waves separately, for a selection of better recorded large shallow earthquakes as listed by Gutenberg and Richter [1954]. Those for which there was suspicion of depth in excess of the normal (believed to be about $25 \mathrm{~km}$ ) were rejected. Values of $m_{B}$ were plotted against those of $M_{S}$, and (1) derived from the plot. The values $a=0.37, b=6.76$ are comparable with those found by Bath [1955] as follows:

\begin{tabular}{llll} 
Station & Body wave used & $\mathrm{a}$ & $\mathrm{b}$ \\
\hline Uppsala & PZ & 0.45 & 6.3 \\
& PH & 0.46 & 6.4 \\
& SH & 0.23 & 5.6 \\
\multirow{5}{*}{ Kiruna } & PZ & & \\
& PH & 0.59 & 6.2 \\
& SH & 0.50 & 6.5 \\
& & 0.30 & 6.1
\end{tabular}

At Pasadena, a weighted mean is taken between $m_{B}$ as found directly from body waves, and $m_{S}$, the corresponding value derived from $M_{S}$ by applying the relation (1), or still better from tables and charts set up to give $m_{S}$ directly from surface wave data. This weighted mean is designated the unified magnitude denoted by $m$.

In Figure 1 residuals $m_{B}-m_{S}$ on the basis $a=0.37$, $b=6.76$ are plotted against $m$, using amplitude and period data from all available station bulletins,

a) for all shocks in Table 13 of Gutenberg and Richter [1954] for which there was no indication of depth exceeding $30 \mathrm{~km}$, excluding all uncertain or doubtful magnitudes;

b) using all similar data for Table 14 of Gutenberg and Richter [1954] for 1936-1939 and 1950-1952 (inclusive). There is little indication of systematic deviation from the axis of zero residuals. The slight apparent excess of positive residuals may be due to the use of a few shocks with depths somewhat greater than supposed, which should result in a 


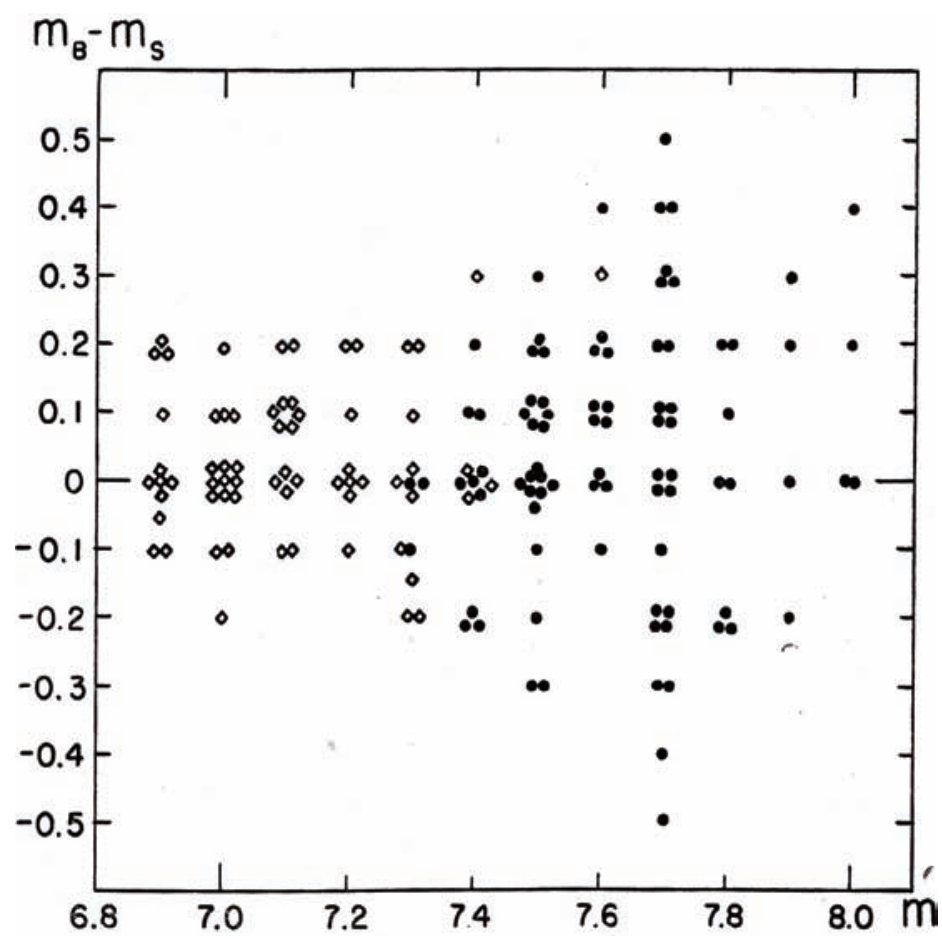

Figure 1. Residuals $m_{B}-m_{S}$ on the basis $a=0.37, b=6.76$ are plotted against $m$, using amplitude and period data from all available station bulletins.

decrease of $m_{S}$.

Comparable data for magnitudes below 7 are rare. Ten of eleven shocks in the California region, with magnitudes near 6 , give $m_{B}-m_{S}$ from +0.1 to -0.2 ; the eleventh gives -0.4 .

Figure 2 is a nomogram prepared by Mr. J.M. Nordquist for the direct determination of $m_{S}$ from surface wave amplitudes. The corresponding values of $M_{S}$ and of $\log E$ from equation (6), are also indicated.

The adjustment of $M_{L}$ to $m$ or $M$ cannot yet be determined so closely as that of $m$ to $M$ but can be stated with an error not likely to exceed 0.5 magnitude unit for those shocks (magnitudes 3 to 6) most often rated in terms of $M_{L}$. Representative results are given in Table 1. Values in parenthesis are outside the observable range.

Until 1954, the writers generally reported magnitudes for large deep shocks, and for large shallow teleseisms as determined from body waves, effectively in terms of $M_{S}$, first determining $m_{B}$ and then correcting to $M_{S}$ by applying equation (1) or an earlier approximation to it. The correction was usually applied only to shocks of magnitude 7 or over.

It now develops [Gutenberg and Richter 1956] that many outstanding difficulties disappear if the linear relation

\begin{tabular}{|c|c|c|c|c|c|c|c|}
\hline $\mathrm{ML}_{\mathrm{L}}$ & 3 & 4 & 5 & 6 & 7 & 8 & 9 \\
\hline $\mathrm{M}$ & $(2.4)$ & $(3.6)$ & 4.7 & 5.8 & 6.8 & 7.9 & $(8.9)$ \\
\hline $\mathrm{m}$ & $(4.0)$ & $(4.7)$ & 5.4 & 6.1 & 6.8 & 7.5 & $(8.1)$ \\
\hline $\log \mathrm{E}$ & 15.4 & 17.2 & 18.9 & 20.5 & 22.1 & 23.7 & $(25.2)$ \\
\hline
\end{tabular}

Table 1. Values of $M, m$ and $\log E$ for given values of $M_{L}$, using $M=1.27\left(M_{L}-1\right)-0.016 M_{L}^{2}, m=0.63 M+2.5, \log E=5.8+2.4 m(E=$ energy in ergs).
(2) is consistently extended to magnitudes below 7 . Whereas shocks of the largest magnitude record with surface waves relatively large compared with the body waves, shocks of magnitude below 7 show relatively small surface waves when recorded at teleseismic distances. Many long-period instruments do not record such shocks clearly; this makes assignment of magnitude from the data of distant stations difficult. Short-period instruments in such cases may show a measurable $P$; the absence of recorded surface waves is then sometimes misinterpreted as evidence for deep focus.

When equation (2) is used, and data for both body waves and surface waves are available, two different determinations are in effect available for either $m$ or $M$. The equation gives $m_{B}=M_{S}$ for a value near $63 / 4$. When the magnitude does not greatly differ from this figure, problems of adjustment are minor, and reduce to judgement as to the relative reliability of the two groups of data.

Although at present many more stations report amplitudes for surface waves than for body waves, some ten years' experience indicates that $m_{B}$ provides the better data in practice as shown by fewer systematic errors and more consistent results, as well as being theoretically preferable.

In using station bulletins to determine $M_{S}$, the maxima of surface waves can be used for magnitude only when the period is near 20 seconds. If the period is not specified, there is risk that the reported maximum amplitude may refer to much longer or shorter waves, which seriously falsifies $M_{S}$. With some exceptions, magnitudes currently being reported in station bulletins are either $M_{L}$ determined from nearby stations, or $M_{S}$. There is less general determination of $m_{B}$, and the relation in equation (1) or (2) is often overlooked. Occasionally $M_{S}$ is even given for deep shocks as found directly from surface waves; if the hypocenter is deeper than about $30 \mathrm{~km}$, calculation on this basis gives too low a value.

Routine station bulletins issued from Pasadena continue to list magnitudes $M$ which are either $M_{L}$ or $M_{S}$; but beginning with 1954 the annual list of large shocks also tabulates $m$, which is an intermediate step toward a definitive magnitude-energy relation.

The practical definition of the unified magnitude $m$ consists in a system of tables and charts for calculating magnitude from the quotient amplitude/period for the maximum waves of the principal wave groups $P, P P$, and $S$. This quotient is used in the form

$$
q=\log u / T \text { or } q=\log w / T
$$

where $u$ and $w$ are respectively the horizontal and vertical components of the ground displacements in microns and $T$ the period in seconds. Each table or chart gives for all distances and focal depths a quantity $Q$ such that for corresponding distance and depth

$$
m=q+Q+s
$$


EARTHQUAKE MAGNITUDE AND ENERGY

CALCULATED FROM MAXIMUM GROUND AMPLITUDE IN MICRONS (COMBINED HORIZONTAL COMPONENTS) FOR SURFACE WAVES OF 20-SECOND PERIOD.

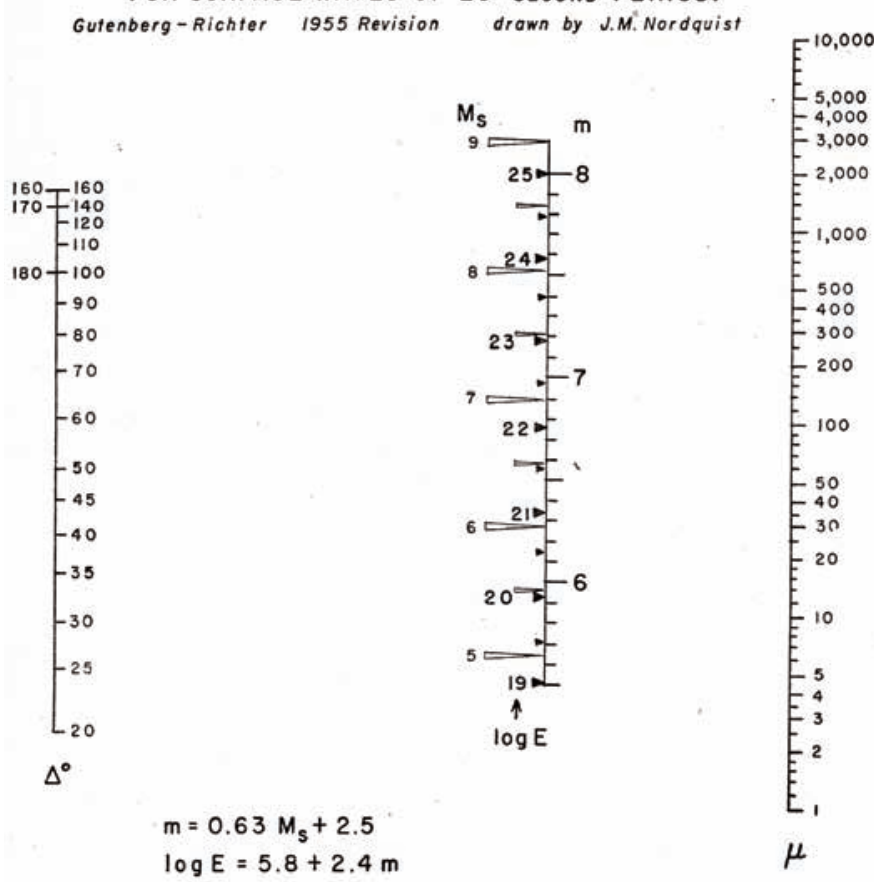

Figure 2. Nomogram for the direct determination of $m_{S}$ from surface wave amplitudes. The corresponding values of $M_{S}$ and of $\log E$ from equation (6) are also indicated.

where $s$ is a ground correction characteristic of the station used.

Charts and tables of this type were first given by Gutenberg [1945b, 1945c], where $Q$ was designated $A$. Those accompanying the present paper (Table 2, Figures 3, 4 and 5) represent no change in fundamental concept, but only a revision. The statistical processes by which the tables and charts published in 1945 were derived have now been repeated by the senior author using a much larger body of data, and, it is hoped, with greater precision. One effect has been to remove a persistent discrepancy between magnitudes determined from horizontal and vertical components; this discrepancy was discovered independently by Bath [1955].

This procedure places the unified magnitude $m$ on a selfconsistent and independent basis as satisfactory for teleseisms as that of $M_{L}$ for local earthquakes, and with the great advantage of being applicable directly to seismograms recorded on instruments of all types and at all stations. If desired, a formal definition for $m$ may be phrased as follows:

$$
m-7.0=q n
$$

at a distance of $90^{\circ}$ for normal shallow focal depth, where $q=\log w / T$ refers to $P Z$, and the station constant $s$ is taken as zero, representing average station ground conditions.

Since the relation of $M_{L}$ to $m$ is not yet on a definitive basis, the authors suggest that the «Richter scale» as defined in 1935 be retained for determining magnitudes of local

\begin{tabular}{|c|c|c|c|c|c|c|c|c|c|c|c|c|c|c|c|c|c|}
\hline \multicolumn{6}{|c|}{$\triangle \mathrm{PZ}$ PH PPZ PPH SH } & \multicolumn{6}{|c|}{$\Delta \mathrm{PZ}$ PH PPZ PPH SH } & \multicolumn{6}{|c|}{$\triangle \mathrm{PZ}$ PH PPZ PPH SH } \\
\hline 16 & 59 & 60 & & & 72 & 56 & 68 & 71 & 69 & 70 & 66 & 96 & 73 & 76 & 72 & 74 & 71 \\
\hline 17 & 59 & 60 & & & 68 & 57 & 68 & 71 & 69 & 70 & 66 & 97 & 74 & 78 & 72 & 74 & 72 \\
\hline 18 & 59 & 60 & & & 62 & 58 & 68 & 71 & 70 & 71 & 66 & 98 & 75 & 78 & 72 & 74 & 73 \\
\hline 19 & 60 & 61 & & & 58 & 59 & 68 & 71 & 70 & 72 & 66 & 99 & 75 & 78 & 72 & 74 & 73 \\
\hline 20 & 60 & 61 & & & 58 & 60 & 68 & 71 & 71 & 73 & 66 & 100 & 74 & 77 & 72 & 74 & 74 \\
\hline 21 & 61 & 62 & & & 60 & 61 & 69 & 72 & 72 & 74 & 67 & 101 & 73 & 76 & 72 & 74 & 74 \\
\hline 22 & 62 & 63 & & & 62 & 62 & 70 & 73 & 73 & 74 & 67 & 102 & 74 & 77 & 72 & 74 & 74 \\
\hline 23 & 63 & 64 & & & 62 & 63 & 69 & 73 & 73 & 74 & 67 & 103 & 75 & 79 & 72 & 74 & 73 \\
\hline 24 & 63 & 65 & & & 62 & 64 & 70 & 73 & 73 & 75 & 68 & 104 & 76 & 79 & 73 & 75 & 73 \\
\hline 25 & 65 & 66 & & & 62 & 65 & 70 & 74 & 73 & 75 & 69 & 105 & 77 & 81 & 73 & 75 & 72 \\
\hline 26 & 64 & 66 & & & 62 & 66 & 70 & 74 & 73 & 74 & 69 & 106 & 78 & 82 & 74 & 76 & 72 \\
\hline 27 & 65 & 67 & & & 63 & 67 & 70 & 74 & 72 & 74 & 69 & 107 & 79 & 83 & 74 & 76 & 72 \\
\hline 28 & 66 & 67 & & & 63 & 68 & 70 & 74 & 71 & 73 & 69 & 108 & 79 & 83 & 74 & 76 & 72 \\
\hline 29 & 66 & 67 & & & 63 & 69 & 70 & 74 & 70 & 72 & 69 & 109 & 80 & 84 & 74 & 76 & 72 \\
\hline 30 & 66 & 68 & 67 & 68 & 63 & 70 & 69 & 73 & 70 & 72 & 69 & 110 & 81 & 85 & 74 & 76 & 72 \\
\hline 31 & 67 & 69 & 67 & 68 & 63 & 71 & 69 & 73 & 71 & 73 & 70 & 112 & 82 & 86 & 74 & 76 & \\
\hline 32 & 67 & 69 & 68 & 69 & 64 & 72 & 69 & 73 & 71 & 73 & 70 & 114 & 86 & 90 & 75 & 77 & \\
\hline 33 & 67 & 69 & 68 & 69 & 64 & 73 & 69 & 72 & 71 & 73 & 69 & 116 & 88 & & 75 & 77 & \\
\hline 34 & 67 & 69 & 68 & 69 & 65 & 74 & 68 & 71 & 70 & 72 & 68 & 118 & 90 & & 75 & 77 & \\
\hline 35 & 67 & 69 & 68 & 69 & 66 & 75 & 68 & 71 & 69 & 71 & 68 & 120 & & & 75 & 77 & \\
\hline 36 & 66 & 68 & 67 & 68 & 66 & 76 & 69 & 72 & 69 & 71 & 68 & 122 & & & 74 & 76 & \\
\hline 37 & 65 & 67 & 67 & 68 & 66 & 77 & 69 & 72 & 69 & 71 & 68 & 124 & & & 73 & 75 & \\
\hline 38 & 65 & 67 & 67 & 68 & 66 & 78 & 69 & 73 & 69 & 71 & 69 & 126 & & & 72 & 74 & \\
\hline 39 & 64 & 66 & 66 & 67 & 67 & 79 & 68 & 72 & 69 & 71 & 68 & 128 & & & 71 & 74 & \\
\hline 40 & 64 & 66 & 66 & 67 & 67 & 80 & 67 & 71 & 69 & 71 & 67 & 130 & & & 70 & 73 & \\
\hline 41 & 65 & 67 & 65 & 66 & 66 & 81 & 68 & 72 & 70 & 72 & 68 & 132 & & & 70 & 73 & \\
\hline 42 & 65 & 67 & 65 & 66 & 65 & 82 & 69 & 72 & 71 & 73 & 69 & 134 & & & 69 & 72 & \\
\hline 43 & 65 & 67 & 66 & 67 & 65 & 83 & 70 & 74 & 72 & 74 & 69 & 136 & & & 69 & 72 & \\
\hline 44 & 65 & 67 & 67 & 68 & 65 & 84 & 70 & 74 & 73 & 75 & 69 & 138 & & & 70 & 73 & \\
\hline 45 & 67 & 69 & 67 & 68 & 65 & 85 & 70 & 74 & 73 & 75 & 68 & 140 & & & 71 & 74 & \\
\hline 46 & 68 & 71 & 67 & 68 & 66 & 86 & 69 & 73 & 73 & 75 & 67 & 142 & & & 71 & 74 & \\
\hline 47 & 69 & 72 & 67 & 68 & 66 & 87 & 70 & 73 & 72 & 74 & 68 & 144 & & & 70 & 73 & \\
\hline 48 & 69 & 72 & 67 & 68 & 67 & 88 & 71 & 75 & 72 & 74 & 68 & 146 & & & 69 & 72 & \\
\hline 49 & 68 & 71 & 67 & 68 & 67 & 89 & 70 & 74 & 72 & 74 & 68 & 148 & & & 69 & 72 & \\
\hline 50 & 67 & 70 & 67 & 68 & 66 & 90 & 70 & 73 & 72 & 74 & 68 & 150 & & & 69 & 72 & \\
\hline 51 & 67 & 70 & 67 & 68 & 65 & 91 & 71 & 75 & 72 & 74 & 69 & 152 & & & 69 & 72 & \\
\hline 52 & 67 & 70 & 67 & 68 & 65 & 92 & 71 & 74 & 72 & 74 & 69 & 154 & & & 69 & 72 & \\
\hline 53 & 67 & 70 & 67 & 68 & 66 & 93 & 72 & 75 & 72 & 74 & 69 & 156 & & & 69 & 72 & \\
\hline 54 & 68 & 71 & 68 & 69 & 66 & 94 & 71 & 74 & 72 & 74 & 70 & 158 & & & 69 & 72 & \\
\hline 55 & 68 & 71 & 69 & 70 & 66 & 95 & 72 & 76 & 72 & 74 & 70 & 160 & & & 69 & 72 & \\
\hline & & & & & & & & & & & & 170 & & & 69 & 72 & \\
\hline
\end{tabular}

Table 2. Values of $Q$ for shallow shocks.

shocks. For teleseisms, the use of the unified scale $m$ is preferred and strongly recommended. For magnitudes from about $5 \frac{1}{2}$ to 7 , the departure between the two scales is within the usual limits of error under the now existing conditions of recording and reporting amplitudes. Gutenberg and Richter [1954] have not assigned magnitudes below 6 to shocks outside the California area (within which $M_{L}$ is reported); such shocks are merely designated by the letter $d$. Above magnitude $7 \frac{1}{2}$ the scales diverge significantly; but then determinations from the data of numerous stations scatter increasingly, and it is advisable to distinguish carefully between determinations from body waves and from surface waves. It is urgent that magnitudes determined from seismograms at single stations should not be published unaccompanied by the amplitude and period readings on which they are based.

It is hoped that before many years have passed it will be possible to express the entire range of observed magnitudes in terms of the unified magnitude $m$.

Since the provisional use of $m$ is especially intended for investigations relating to energy, $m$ is being published together with the energy calculated from it by the relation

$$
\log E=5.8+2.4 m
$$

to be established on a later page.

For most types of publication the writers think it preferable to follow a suggestion by Dr. L.B. Slichter, giving the 

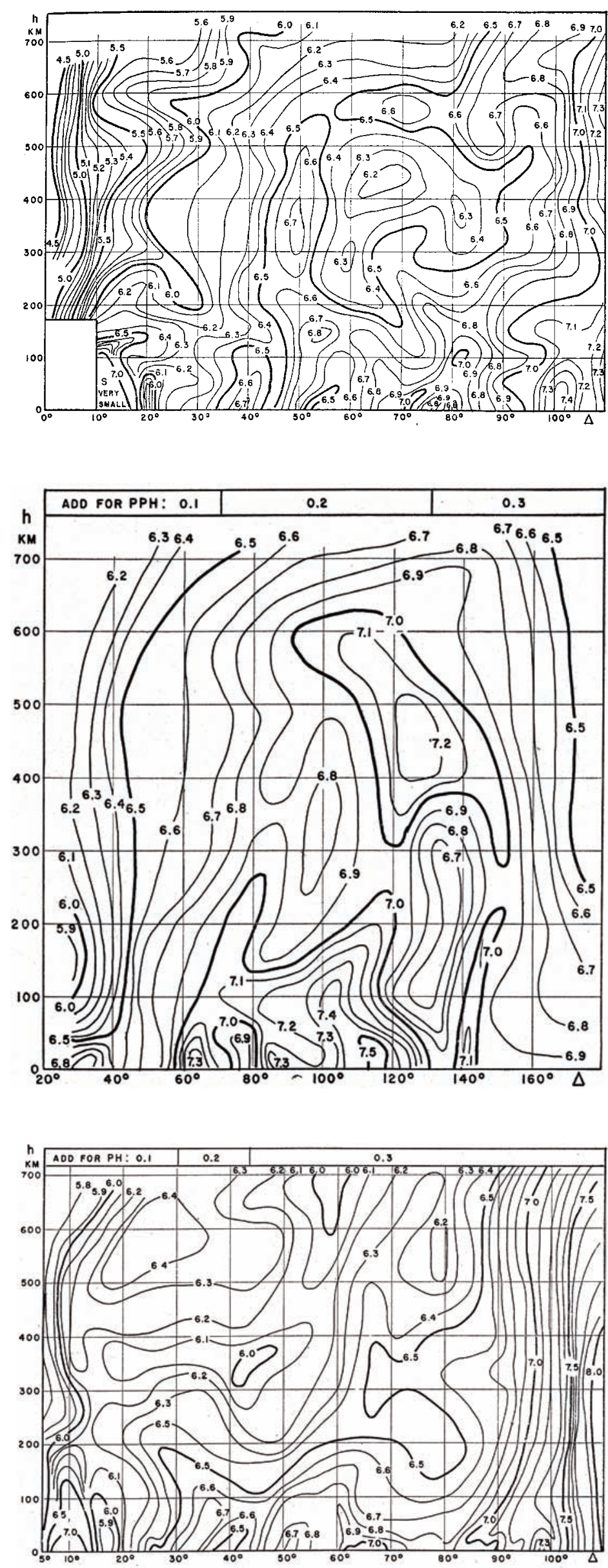

Figures 3, 4, 5. From top: Revised values of $Q$ for $S H$; Revised values for $Q$ for $P P Z$; Revised values for $Q$ for $P Z$. value of $\log E$ together with equation (6), and so avoiding confusion due to use of numerically different magnitude scales.

Most calculations of the magnitude-energy relation depend directly or indirectly on the equation for a wave group from a point source [Gutenberg and Richter 1956]

$$
E=2 \pi^{3} h^{2} v \rho(A / T)^{2} t
$$

where $E$ is energy, $h$ is linear distance from the source, $v$ is velocity, $\rho$ is density, $A$ and $T$ are amplitude and period of sinusoidal waves, and $t$ is the duration of the wave group (which hence contains $n=t / T$ waves).

This applies at the epicenter when $h$ is hypocentral depth, and includes a factor which takes account of the effect of the free surface. Taking $v=3.4 \mathrm{~km} / \mathrm{sec}$ for transverse waves, applying a factor $3 / 2$ to allow for half as much energy in longitudinal waves, and using $h=16 \mathrm{~km}, \rho=2.7 \mathrm{gm} / \mathrm{cc}$, this reduces to

$$
\log E=12.34+2 q_{o}+\log t_{o}
$$

where $q=\log A / T$ and the subscript zero refers to the epicenter. A fundamental empirical equation is

$$
q_{o}=-0.6+0.8 M_{L}-0.01 M_{L}^{2}
$$

This is a revised result drawn from the plot of $q_{0}$ as a function of $M$ for California shocks (Figure 3) [Gutenberg and Richter 1956]. Two further important equations are derived from plotted data (Figures 6):

$$
\log t_{o}=-1+0.4 q_{0}
$$

and

$$
q_{0}=m-2.3
$$

For the latter result most of the data cover a relatively small range of $m$. Combining these

$$
\log t_{o}=0.4 m-1.9
$$

On the other hand, if in (10) we substitute for $q_{o}$ its expression in terms of $M_{L}$ from (9), we obtain

$$
\log t_{o}=-1.24+0.32 M_{L}-0.04 M_{L}^{2}
$$

which differs only slightly from the corresponding equation setup empirically in Gutenberg and Richter [1956], showing that the derivation of (9) and (10) has been consistent.

Combining (9) and (11)

$$
m=1.7+0.8 M_{L}-0.01 M_{L}^{2}
$$

This is drawn on Figure 7; it is not inconsistent with the plotted data. 


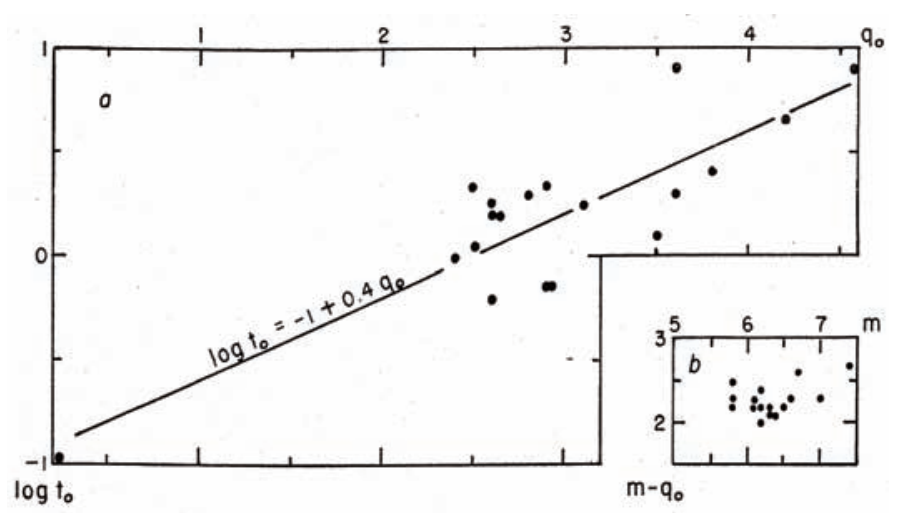

Figure 6. Two important equations are derived from plotted data: $\log t_{o}=-1+0.4 t_{o}$ and $q_{o}=m-2.3$.

If instead of (9) we had used the corresponding equation in Gutenberg and Richter [1956], which has a larger coefficient of the quadratic term, the resulting equation replacing (14) would lead to calculated values of $M_{B}-M_{L}$ which for large $m$ are systematically too small to suit the observations. This is the chief reason for revising the empirical relation between $q_{o}$ and $M_{L}$ to the form (9).

If we apply the relation (2) to (14) we find

$$
M_{S}=1.27\left(M_{L}-1\right)-0.016 M_{L}^{2}
$$

Equation (6) results from substituting in (8) the expressions for $q_{o}$ and $\log t_{0}$ from (11) and (12). It has also been verified approximately by the following calculation. For a train of $n(=t / T)$ sinusoidal body waves, emerging to the surface of the earth at arc distance $\Theta$ from a surface source with horizontal ground displacement $u$, the total energy calculated as radiated from the source is

$$
E=8 \pi^{3} R^{2} \rho v t(u / T)^{2} / U^{2} L
$$

where

$$
U^{2} / f_{i}^{2}=\tan i d i / \sin \Theta d \Theta
$$

Here $E=$ energy, $R=$ radius of the earth, $\rho=$ density, $v=$ velocity, $t=$ duration of wave train, $T=$ period, $i=$ angle of incidence, $f_{i}$ is a factor expressing the effect of the free surface as a function of $i$ (otherwise it depends only on Poisson's ratio; see Gutenberg 1944), and $L$ is a factor to allow for absorption, scattering, internal friction, effects of discontinuities, etc.

There are several simplifying assumptions: the earth is taken as spherically symmetrical, the effect of hypocentral depth is neglected (it is easily corrected for), energy flux is calculated by the ray method as used in geometrical optics, and the use of (16) to calculate total energy implies radiation equally in all directions from the source. In what follows it is assumed that $1 / 3$ of the original energy is radiated as longitudinal waves, and a factor 3 is accordingly applied.

Analogous equations to (17) and (18) apply to the vertical component of ground displacement, replacing $u$ by

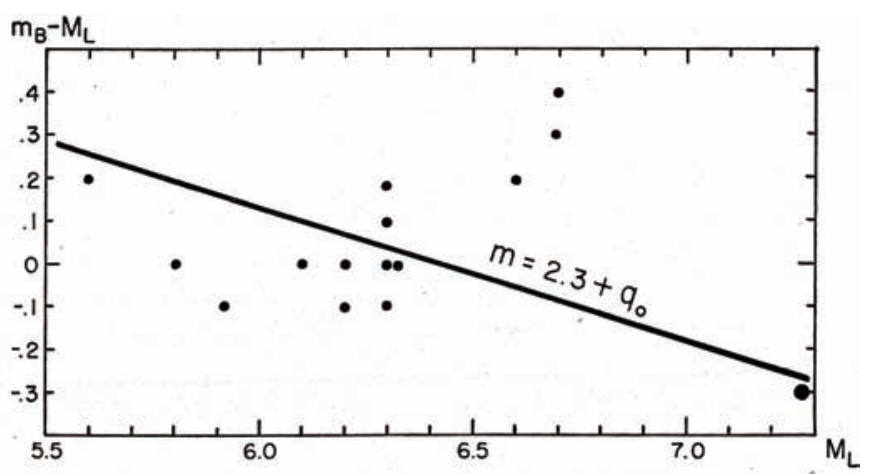

Figure 7. Combining equations (9) and (11), $m=1.7+0.8 M_{L}-0.01 M_{L}{ }^{2}$.

$w$ and $U^{2}$ by a similar factor $W^{2}$.

We next take $\rho=3 \mathrm{gm} / \mathrm{cc}, v=6.3 \mathrm{~km} / \mathrm{sec}$ (applying to longitudinal waves), $R=6370 \mathrm{~km}$; we also take $q=\log u / T$ or $q=\log w / T$, where $u$ and $w$ are expressed in microns. Taking the logarithm of (17) with proper attention to the units used, we arrive at

$$
\log E=18.8+\log t+2 q-\log U-\log L
$$

We now assume that $t=t_{o}$; this has been confirmed roughly by Dr. C. Lomnitz from seismograms recorded at Pasadena. Applying (12) with $t$ in place of $t_{o}$, and putting $q=$ $m-Q$,

$$
\log E=16.9+2.4 m-2 Q-2 \log U-\log L
$$

Comparing this with (6) we should have

$$
2 Q+2 \log U+\log L=11.1
$$

and a similar equation for the vertical component. Here, $2 Q$ may be taken from Table 2, and $\log U$ can be calculated from (17). Working this out for the vertical component of $P$ waves, the following values are found:

$\begin{array}{crrr}\Theta & 20^{\circ} & 42^{\circ} & 100^{\circ} \\ \log L & -2.0 & -1.3 & -1.5\end{array}$

The calculation cannot be extended reliably to distances less than $20^{\circ}$. The contribution of absorption to $\log L$ should be about 0.4 near $100^{\circ}$ and 0.3 at moderate distances. Loss by refraction at the Mohorovicic, Conrad, and other discontinuities in the crust may account for a few tenths in $\log L$. This leaves about one unit in $\log L$ unaccounted for. If all assumptions are correct, energy flux is reduced to roughly one tenth within the first $20^{\circ}$ of distance; this must occur within the upper $200 \mathrm{~km}$ of the mantle. If this is correct, we should expect a smaller constant term in the energymagnitude relation corresponding to (6) for shocks at greater depths. This would agree with the relatively low energy 
calculated by Sagisaka [1954] for a shock at a depth of $360 \mathrm{~km}$. However, the constant term 5.8 in (6) and the coefficient 0.4 in (12) are not accurately fixed, and $\log E$ calculated from (6) may be in error by as much as one unit.

Acknowledgements. Contribution n. 750, Division of the Geological Sciences, California Institute of Technology, Pasadena, California.

\section{References}

Bath, M. (1955). The Problem of Earthquake Magnitude Determination (unpublished).

Gutenberg, B. (1944). Energy Ratio of Reflected and Refracted Seismic Waves, Bull. Seismol. Soc. Amer., 34, 85-102.

Gutenberg, B. (1945a). Amplitudes of Surface Waves and Magnitude of Shallow Earthquakes, Bull. Seismol. Soc. Amer., 35, 3-12.

Gutenberg, B. (1945b). Amplitudes of P, PP, and S and Magnitude of Shallow Earthquakes, Bull. Seismol. Soc. Amer., 35, 57-69.

Gutenberg, B. (1945c). Magnitude Determination for Deep-focus Earthquakes, Bull. Seismol. Soc. Amer., 35, 117-130.

Gutenberg, B., and C.F. Richter (1936). On Seismic Waves (third paper), Gerlands Beitrage zur Geophysik, 47, 73-131.

Gutenberg, B., and C.F. Richter (1942). Earthquake Magnitude, Intensity, Energy and Acceleration, Bull. Seismol. Soc. Amer., 32, 163-191.

Gutenberg, B., and C.F. Richter (1954). Seismicity of the Earth, second ed., Princeton Press.

Gutenberg, B., and C.F. Richter (1956). Earthquake Magnitude, Intensity, Energy and Acceleration (second paper), Bull. Seismol Soc. Amer., 46, 2, 105-145.

Richter, C.F. (1935). An Instrumental Magnitude Scale, Bull. Seismol. Soc. Amer., 25, 1-32.

Sagisaka, K. (1954). On the Energy of Earthquakes, Geophys. Mag. Tokyo, 26, 53-82.

(C) 2010 by the Istituto Nazionale di Geofisica e Vulcanologia. All rights reserved. 